\title{
Neuroprotective Effect of Oral Administration of Trigonella Foenum-Graecum on Chronic Cerebral Hypoperfusion in Rat Model
}

Hulol Saleh Alruhaimi ${ }^{1}$, Ahmed Kaid Allow ${ }^{1}$,Muhammed Naser Bahbah ${ }^{2}$, Zunariah Buyong $^{1}$, Shaikh Mizanur Rahman ${ }^{1}$, Wael Mohamed Yousef ${ }^{1}$

${ }^{1}$ Department of Basic Medical Sciences, Kulliyyah of Medicine, International Islamic University Malaysia

${ }^{2}$ Department of Biology, Faculty of Sciences, Princess Nourah bint Abdulrahman University, Riyadh, Kingdom of Saudi Arabia

Presenter: Hulol Saleh Alruhaimi

Introduction: Decreased cerebral blood supply to the brain can generate a condition of chronic cerebral hypoperfusion, which is one of the pathophysiological mechanisms of neuronal degeneration and cognitive impairment in Alzheimer's disease. This study aimed to evaluate the potential neuroprotective effects of Trigonella foenum graecum seeds on chronic cerebral hypoperfusion in a rat model. Materials and Methods: Chronic cerebral hypoperfusion was induced by permanent bilateral ligation of the common carotid arteries (a two-vessel occlusion, 2VO) in male Sprague-Dawley rats. The experimental groups were divided into three groups (11 rats/group): i) sham (control) group; ii) $2 \mathrm{VO}$ group without any treatment; and iii) $2 \mathrm{VO}$ group that was administered orally with the Trigonella foenum graecum extract $(100 \mathrm{mg} / \mathrm{kg} /$ day) by oral gavage from 3 days before the date of $2 \mathrm{VO}$ surgery and continued daily until the end of the 8th postoperative week. Spatial memory performance was assessed by the Morris water maze test. Malondialdehyde and C-reactive protein (CRP) concentration, superoxide dismutase and glutathione activities were measured in serum after 8 weeks from 2VO injury. Results: Chronic cerebral hypoperfusion rats resulted in spatial memory impairments. This behavioral dysfunction was accompanied by decreasing superoxide dismutase and glutathione activities, and increasing malondialdehyde and CRP levels in serum. Oral administration of Trigonella foenum graecum extract significantly improved the memory impairment, enhanced antioxidant enzyme activities, and decreased the malondialdehyde and CRP levels to near normal levels. Conclusion: The potential activity offered by Trigonella foenum graecum extract showed the neuroprotective effect that may be beneficial in cerebrovascular type dementia. 